\title{
Algorithm for preparation of multilayer systems with high critical angle of total reflection
}

\author{
I. Carron \\ CSCE, Texas A\&M University, College Station, Texas 77843 \\ V. Ignatovich \\ FLNP JINR, Dubna Moscow Region, 141980, Russia \\ (Received 6 December 2002; published 22 April 2003)
}

\begin{abstract}
A development of the theory of multilayer systems is presented. It shows precisely how to calculate thicknesses and number of layers to get reflectivity close to unity for a given arbitrary critical angle. Application of the proposed approach to real systems is demonstrated.

DOI: 10.1103/PhysRevA.67.043610
\end{abstract}

\section{INTRODUCTION}

We apply an analytical method not yet widely known to the calculation of thicknesses and number of layers in multilayer systems (MS) in order to achieve a high critical angle.

Usually MS consist of many bilayers of two materials with different refraction indices, and the thickness $a_{j}$ of the bilayer varies with its index $j$. The idea to increase the critical angle with the help of MS with steadily decreasing thickness of layers was first published by Turchin [1] in 1967 and further elaborated by the author in 1997 [2]. In the meanwhile another contribution by Mezei, dealing with the same subject, appeared in the literature in 1976 [3].

The main question is what should be the rule of thickness variation. At present, the thickness is varied according to the theoretical prescriptions as described in Ref. [4]. All the bilayers have different thickness, and the change of the thickness of neighboring layers can be very small.

We consider here a different construction: the MS consist of several periodic chains, and we show how to find the period, number of periods for every chain, and the number of chains to achieve the critical angle we wish. We use a recurrence method similar to that used by Darwin [5] and later by Godfrey [6]. Our method is analytical. It permits us to control technology of layers preparation, to find the optimal number of layers for achieving a tolerable result, and to repair defects, if for some reasons they appear on the reflection curve.

Applications of MS in experiments are discussed in many review papers (see, for instance Refs. [7,8] and references therein), and we do not dwell on it too much. We only want to add some references [9-15], which were not mentioned in Ref. [8].

In Refs. [9-12] the MS were used for the polarization of neutrons by transmission [9] through them, by transportation along magnetized neutron guides [10,12], and by splitting of an unpolarized beam by a magnetized supermirror [11]. In Ref. [13] the pulsed beam was produced by reflection from a supermirror periodically magnetized in an external field. In Ref. [14] supermirrors were used in neutron guides to increase the transmitted flux. Some research on fabrication of supermirrors was presented in Ref. [15].

Our present paper stems from a desire to increase the intensity and polarization of polarized neutron beams. In or- der to increase intensity we need to increase critical angle $\theta_{+}$ for one component of polarization. In order to increase polarization we need to decrease critical angle $\theta_{-}$for the opposite component of polarization. However, in this paper we do not mention polarization since our method is more general. This method can be applied to MS, which contain more than two materials, and it can be applied to other radiation such as $\mathrm{x}$ rays, which have polarization properties different from those of neutrons. As for the specific problems of neutron polarization, they can be solved by an appropriate choice of materials comprising a multilayer system.

\section{OUR METHOD}

First of all, let us mention one difference between our approach and the one commonly used. We consider reflection in terms of the normal component $k_{\perp}$ of the incident neutron wave vector instead of the incidence angle. This is more convenient because reflection of a mirror at a given angle depends also on the wavelength, whereas when it is stated in terms of the wave vector $\boldsymbol{k}$ it depends only on $k_{\perp}$ and properties of the mirror. In the following development, we even omit the index $\perp$, and use simply $k$, because we deal only with specular reflection and for this case, the one dimension is sufficient.

To be more precise we consider a neutron propagating along the $x$ axis normal to the supermirror and calculate its reflection from a set of alternating layers of two materials. One of them is represented by a potential barrier of height $u_{b}$ and width $l_{b}$, another is represented by a potential well of height $u_{w}$ and width $l_{w}$. The potential barrier with $l_{b} \rightarrow \infty$ totally reflects neutrons with $k^{2}<u_{b}$, and $\sqrt{u_{b}}$ is called critical number $k_{c}$.

In order for our readers to understand our argument, we first need to specify our units. When we speak about potential energy $u$, we mean that in common units this energy is $V=\hbar^{2} u / 2 m$, where $\hbar$ is the reduced Planck constant $h / 2 \pi$, and $m$ is the neutron mass. The neutron kinetic energy is $E$ $=\hbar^{2} k^{2} / 2 m$. So the reflection from the potential $V_{h}$ $=\hbar^{2} u_{b} / 2 m$ is total when $E<V_{b}$, which is equivalent to $k^{2}$ $<u_{b}$. Critical energy is defined as $E_{c}=V_{b}$, which is equivalent to the definition of the critical wave number $k_{c}^{2}=u_{b}$.

It is convenient to use $1 / k_{c}$ as a unit of length, hence all the variables with dimension of length can be measured in units $1 / k_{c}$, and are made dimensionless from there on. The 


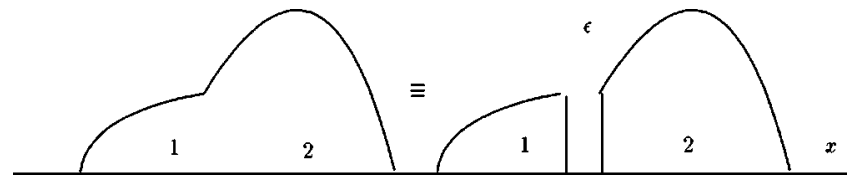

FIG. 1. Every potential can be split by an infinitesimal gap of width $\epsilon \rightarrow 0$ into two. The splitting does not change their reflection and transmission properties, because of total transmission of the gap.

potential barrier becomes of height $u_{b}=1$, and the critical number is unity.

Below we use a somewhat different normalization. We take for unity the difference $u_{b}-u_{w}$, and for the unit length $1 / \sqrt{u_{b}-u_{w}}$. This normalization becomes identical to the one above, when potential of the well is zero, which can be assumed without loss of generality.

We look for such MS that give total reflection up to some $K_{c}>k_{c}=1$. In general, $K_{c}$ can be arbitrarily large, but practically it is not possible to achieve $K_{c}$ larger than 4 .

Our analytical method is based on an observation $[16,17]$ that every potential can be split by an infinitesimal gap into two as shown in Fig. 1, and the reflection amplitude $R_{12}$ of the composite potential is represented as the combination of reflections $R_{i}$ and transmissions $T_{i}$ amplitudes of the separate barriers:

$$
R_{12}=R_{1}+T_{1}^{2} \frac{R_{2}}{1-R_{1} R_{2}},
$$

where the denominator corresponds to multiple reflections inside the gap. For simplicity, in Eq. (1) we did not take into account the asymmetry of the potentials, but it will be discussed later in this paper.

Equation (1) leads immediately to the result [18] obtained for a semi-infinite periodic potential. If a single period of the potential is characterized by reflection and transmission amplitudes $r$ and $t$ respectively, then reflection amplitude of the whole potential (denoted $R_{0}$ in Ref. [4]: Eqs. (14)-(16) there) is

$$
R=\frac{\sqrt{(1+r)^{2}-t^{2}}-\sqrt{(1-r)^{2}-t^{2}}}{\sqrt{(1+r)^{2}-t^{2}}+\sqrt{(1-r)^{2}-t^{2}}}
$$

and the Bloch phase factor [denoted by $\kappa$ in Ref. [4]: Eqs. (12) and (13) there] is

$$
\exp (i q a)=\frac{\sqrt{(1+t)^{2}-r^{2}}-\sqrt{(1-t)^{2}-r^{2}}}{\sqrt{(1+t)^{2}-r^{2}}+\sqrt{(1-t)^{2}-r^{2}}},
$$

where $a$ is the period width and $q$ is the Bloch wave number. At Bragg reflection, $R=\exp (i \chi)$ and $\exp (i q a)=\exp (i \pi n$ $-q^{\prime} a$ ) with real $\chi, q^{\prime}$, and integer $n$. (We neglect here the imaginary part of the potential.)

With Eqs. (2) and (3) we can find [16] reflection $R_{N}$ and transmission $T_{N}$ amplitudes of the periodic chain with a finite number $N$ of periods:

$$
R_{N}=R \frac{1-\exp (2 i q a N)}{1-R^{2} \exp (2 i q a N)},
$$

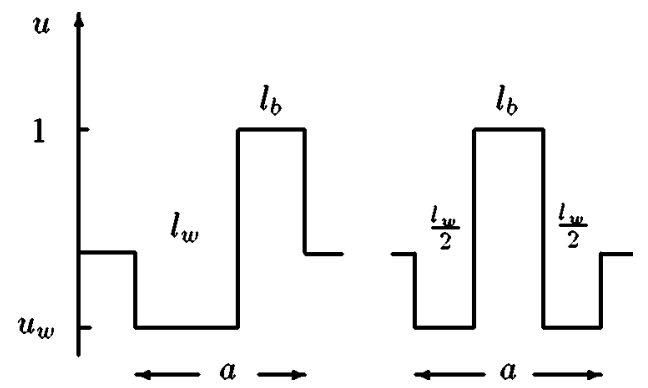

FIG. 2. A period containing a well and a barrier (can be rearranged to a symmetrical form).

$$
T_{N}=\exp (i q a N) \frac{1-R^{2}}{1-R^{2} \exp (2 i q a N)} .
$$

To see how do these formulas work we need to define the single period and its amplitudes $r$ and $t$. A single period is a bilayer. It consists of a potential well and barrier. This period is nonsymmetrical, but we can make it symmetrical by shifting the barrier as shown in Fig. 2. This rearrangement, as we see later, does not change the final result, but it facilitates our mathematics. For a symmetrical period of width $a=l_{w}+l_{b}$ we can immediately find amplitudes $r$ and $t$ :

$$
\begin{gathered}
r=e^{i k_{w} l_{w}} r_{w b} \frac{1-\exp \left(2 i k_{b} l_{b}\right)}{1-r_{w b}^{2} \exp \left(2 i k_{b} l_{b}\right)}, \\
t=e^{i k_{w} l_{w}} e^{i k_{b} l_{b}} \frac{1-r_{w b}^{2}}{1-r_{w b}^{2} \exp \left(2 i k_{b} l_{b}\right)},
\end{gathered}
$$

where $k_{w, b}=\sqrt{k^{2}-u_{w, b}}, r_{w b}=\left(k_{w}-k_{b}\right) /\left(k_{w}+k_{b}\right)$, and potentials may contain an imaginary part accounting for losses.

Substitution of Eq. (5) into Eqs. (2) and (4) gives the result shown in Figs. 3 and 4. In Fig. 3 we see the Bragg reflection with unit amplitude in the interval $\Delta$ called the width of the Darwin table. By decreasing $l_{w}$ and $l_{b}$, we can shift the interval $\Delta$ toward larger $k$, and, if we can built a system of semi-infinite potentials with different periods in

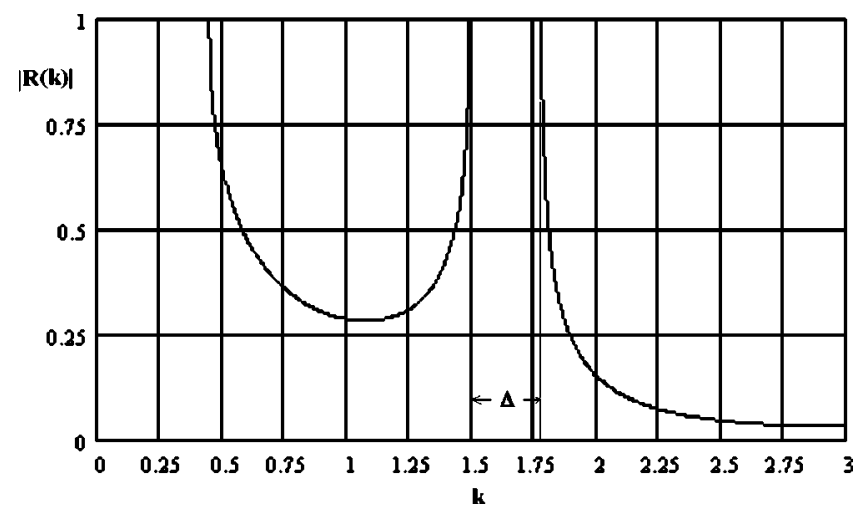

FIG. 3. Reflection amplitude $|R(k)|$ of a semi-infinite periodic potential with period containing the potential well of height $u_{w}$ $=-0.5$ and width $l_{w}=1$, and the barrier of the height $u_{b}=1$ and width $l_{b}=1$. 


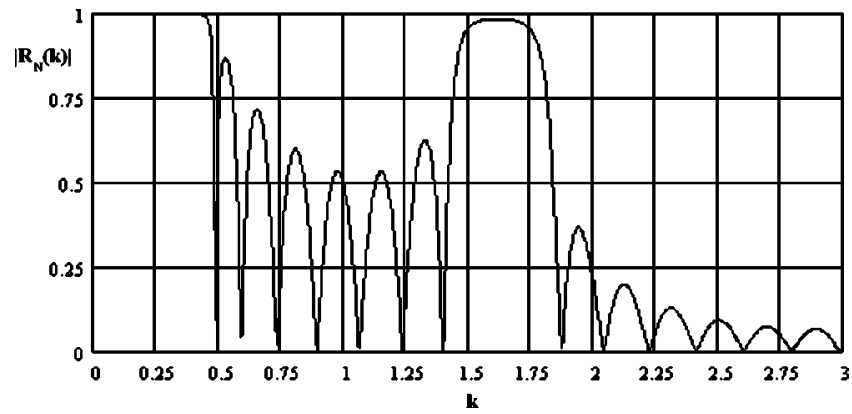

FIG. 4. Reflection amplitude $\left|R_{N}\right|$ of the periodic potential with $N=8$ periods. The parameters of a single period are the same as in Fig. 3.

such a way that intervals $\Delta$ would overlap as intervals $D$ in Fig. 5, we can considerably increase $k_{c}$.

However, we can build periodic chains only with finite number of periods, so we must use $\left|R_{N}\right|$ of Eq. (4), which at the Darwin table is smaller than unity because of $\exp \left(-2 q^{\prime} N a\right)$ in the nominator. This factor is small when $N$ is large. If we tolerate reflection $\left|R_{N}\right|=1-\zeta$ with some small $\zeta$, we must have

$$
N=-\frac{\ln \zeta}{2 a q^{\prime}} .
$$

So the strategy is very clear. We cover step by step the range of $k$ we needed, by overlapping intervals $\Delta^{\prime}<\Delta$, and tuning parameters $l_{w}, l_{b}, N$ we find maximal $\Delta^{\prime}$ needed to minimize the number of required chains, and therefore the total number of layers for the tolerable deviation of reflection coefficient $|R|^{2}$ from unity.

To proceed further it is more convenient to transform Eqs. (2) and (3) to the form

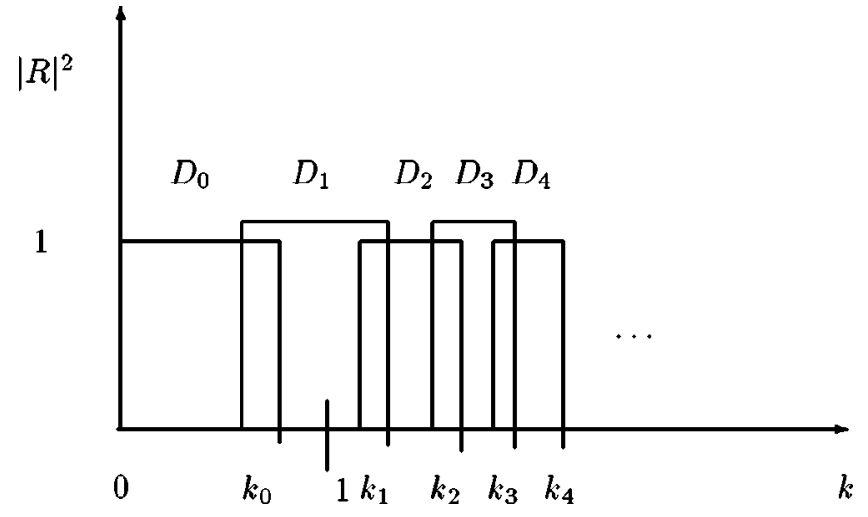

FIG. 5. The algorithm for critical angle increasing. A system of periodic potentials with overlapping Bragg peaks of widths $D_{i}$ gives total reflection in a range of $k$ considerably wider than the common case $0 \leqslant k \leqslant 1$.

$$
\begin{aligned}
R & =\frac{\sqrt{\cos \phi+|r|}-\sqrt{\cos \phi-|r|}}{\sqrt{\cos \phi+|r|}+\sqrt{\cos \phi-|r|}} \\
& =\frac{\sqrt{\operatorname{Re}(r)+|r|^{2}}-\sqrt{\operatorname{Re}(r)-|r|^{2}}}{\sqrt{\operatorname{Re}(r)+|r|^{2}}+\sqrt{\operatorname{Re}(r)-|r|^{2}}}, \\
e^{i q a} & =\frac{\sqrt{\operatorname{Re}(t)+|t|^{2}}-\sqrt{\operatorname{Re}(t)-|t|^{2}}}{\sqrt{\operatorname{Re}(t)+|t|^{2}}+\sqrt{\operatorname{Re}(t)-|t|^{2}}},
\end{aligned}
$$

where $\phi$ is the phase and $\operatorname{Re}(r, t)$ are real parts of amplitudes $r, t$ respectively. To derive Eqs. (7) and (8) we use the relations valid for arbitrary real potential $[18,19]$ :

$$
r=e^{i \phi}|r|, \quad t= \pm i e^{i \phi}|t|, \quad r^{2}-t^{2}=e^{2 i \phi} .
$$

From Eq. (7) it follows that $R$ is a unit complex number $\exp (i \chi)$, when $|r|^{2}>|\operatorname{Re}(r)|$.

\section{ALGORITHM FOR CALCULATIONS OF PARAMETERS OF PERIODIC CHAINS}

Now we show how to calculate $l_{b}, l_{w}, N$, and $\Delta^{\prime}$ for a single chain. Substitution of Eq. (5) into Eqs. (7) and (8) in the case $k^{2}>u_{b}$ gives

$$
R=\frac{\sqrt{\frac{k_{b} \tan \left(k_{w} l_{w} / 2\right)-k_{w} \cot \left(k_{b} l_{b} / 2\right)}{k_{w} \tan \left(k_{w} l_{w} / 2\right)-k_{b} \cot \left(k_{b} l_{b} / 2\right)}}-\sqrt{\frac{k_{w} \tan \left(k_{w} l_{w} / 2\right)+k_{b} \tan \left(k_{b} l_{b} / 2\right)}{k_{b} \tan \left(k_{w} l_{w} / 2\right)+k_{w} \tan \left(k_{b} l_{b} / 2\right)}}}{\sqrt{\frac{k_{b} \tan \left(k_{w} l_{w} / 2\right)-k_{w} \cot \left(k_{b} l_{b} / 2\right)}{k_{w} \tan \left(k_{w} l_{w} / 2\right)-k_{b} \cot \left(k_{b} l_{b} / 2\right)}}+\sqrt{\frac{k_{w} \tan \left(k_{w} l_{w} / 2\right)+k_{b} \tan \left(k_{b} l_{b} / 2\right)}{k_{b} \tan \left(k_{w} l_{w} / 2\right)+k_{w} \tan \left(k_{b} l_{b} / 2\right)}}}
$$

or

$$
R=\frac{\sqrt{\frac{\cos \phi_{+}+r_{w b} \cos \phi_{-}}{\cos \phi_{+}-r_{w b} \cos \phi_{-}}}-\sqrt{\frac{\sin \phi_{+}+r_{w b} \sin \phi_{-}}{\sin \phi_{+}-r_{w b} \sin \phi_{-}}}}{\sqrt{\frac{\cos \phi_{+}+r_{w b} \cos \phi_{-}}{\cos \phi_{+}-r_{w b} \cos \phi_{-}}}+\sqrt{\frac{\sin \phi_{+}+r_{w b} \sin \phi_{-}}{\sin \phi_{+}-r_{w b} \sin \phi_{-}}}},
$$

and 


$$
e^{i q a}=\frac{\sqrt{\cos ^{2} \phi_{+}-r_{w b}^{2} \cos ^{2} \phi_{-}}-\sqrt{-\sin ^{2} \phi_{+}+r_{w b}^{2} \sin ^{2} \phi_{-}}}{\sqrt{\cos ^{2} \phi_{+}-r_{w b}^{2} \cos ^{2} \phi_{-}}+\sqrt{-\sin ^{2} \phi_{+}+r_{w b}^{2} \sin ^{2} \phi_{-}}},
$$

where $\phi_{ \pm}=\left(k_{w} l_{w} \pm k_{b} l_{b}\right) / 2$. It is easy to check that at the limit $l_{b} \rightarrow 0$ we obtain $R \rightarrow 0$ and $q \rightarrow k_{w}$, and at $l_{w} \rightarrow 0$ we obtain $R \rightarrow r_{w b}, q \rightarrow k_{b}$.

If $k^{2}<u_{b}$, instead of Eqs. (11) and (12) we obtain

$$
\begin{aligned}
& R=\frac{\sqrt{\cos ^{2} \xi_{-}-\exp \left(-2 k_{b}^{\prime} l_{b}\right) \cos ^{2} \xi_{+}}-i \sqrt{\sin ^{2} \xi_{-}-\exp \left(-2 k_{b}^{\prime} l_{b}\right) \sin ^{2} \xi_{+}}}{\sqrt{\cos ^{2} \xi_{-}-\exp \left(-2 k_{b}^{\prime} l_{b}\right) \cos ^{2} \xi_{+}}+i \sqrt{\sin ^{2} \xi_{-}-\exp \left(-2 k_{b}^{\prime} l_{b}\right) \sin ^{2} \xi_{+}}}, \\
& e^{i q a}=\frac{\sqrt{\frac{\sin \xi_{-}-\exp \left(-k_{b}^{\prime} l_{b}\right) \sin \xi_{+}}{\sin \xi_{-}+\exp \left(-k_{b}^{\prime} l_{b}\right) \sin \xi_{+}}}-\sqrt{\frac{\cos \xi_{-}-\exp \left(-k_{b}^{\prime} l_{b}\right) \cos \xi_{+}}{\cos \xi_{-}+\exp \left(-k_{b}^{\prime} l_{b}\right) \cos \xi_{+}}}}{\sqrt{\frac{\sin \xi_{-}-\exp \left(-k_{b}^{\prime} l_{b}\right) \sin \xi_{+}}{\sin \xi_{-}+\exp \left(-k_{b}^{\prime} l_{b}\right) \sin \xi_{+}}}+\sqrt{\frac{\cos \xi_{-}-\exp \left(-k_{b}^{\prime} l_{b}\right) \cos \xi_{+}}{\cos \xi_{-}+\exp \left(-k_{b}^{\prime} l_{b}\right) \cos \xi_{+}}}},
\end{aligned}
$$

where $\xi_{ \pm}=k_{w} l_{w} / 2 \pm \phi_{0}, \phi_{0}=\arccos \left(k_{w} / \sqrt{u_{b}-u_{w}}\right)$, and $k_{b}^{\prime}$ $=\sqrt{u_{b}-k^{2}}$.

It is easy to check that in the limit $l_{w} \rightarrow 0$ the periodic potential degenerates to a potential step and we obtain $R$ $\rightarrow r_{w b}=\exp \left(-2 i \phi_{0}\right), q=i k^{\prime}$. In the limit $l_{b} \rightarrow 0$ barriers disappear and we obtain empty space with $R=0$ and $q=k_{w}$.

Now we consider $k^{2}>u_{b}$. The Bragg reflections take place when expressions under two square roots in Eq. (11) have opposite signs. It happens when $\left|\cos \phi_{+}\right|<r_{w b}\left|\cos \phi_{-}\right|$, or $\left|\sin \phi_{+}\right|<r_{w b}\left|\sin \phi_{-}\right|$, i.e., for $\pi n / 2-\delta \phi \leqslant \phi_{+} \leqslant \pi n / 2$ $+\delta \phi$, where $n$ is integer. The half-width of the Bragg reflection ( $\equiv$ half-width of the Darwin table, $\Delta / 2$ ) is $\delta \phi$ $=r_{w b}\left|\cos \phi_{-}\right|$for odd $n$, and $\delta \phi=r_{w b}\left|\sin \phi_{-}\right|$for even $n$. To get this width maximal we must have $\phi_{-}=\pi m / 2$ with integer $m$, and $m$ must be even or odd for odd or even $n$, respectively.

From these considerations we obtain that, if we want to have the total reflection at some $k=k_{v}$, we must require at this point $k_{b} l_{b}+k_{w} l_{w}=\pi$ and $k_{w} l_{w}-k_{b} l_{b}=0$, which immediately gives two parameters $l_{b}=\pi / 2 k_{b}$ and $l_{w}=\pi / 2 k_{w}$ [as was correctly used in Ref. [4], Eq. (7)]. Of course, we can also require $k_{b} l_{b}+k_{w} l_{w}=2 \pi$ and $k_{w} l_{w}-k_{b} l_{b}=\pi$, then we find other parameters $l_{b}=\pi / 2 k_{b}$ and $l_{w}=3 \pi / 2 k_{w}$; however this choice of parameters, as will be shown later, is not profitable.

We cannot use the full width $\Delta$ of the Darwin table, because the total reflection inside it is possible only for an infinite number of periods. With finite number of periods we have some reduction of reflection coefficient, and the reduction is tolerable in some interval $\Delta^{\prime}<\Delta$. The larger is the number $N$ of periods, the wider is $\Delta^{\prime}$. However, the gain in width decreases with increase of $N$. So we need to find an optimal $N$ that gives the maximal effective length $\delta_{1}$ $=\Delta^{\prime} / N$ per single period.

For optimization we represent Eq. (12) at the Bragg peak in the form

$$
e^{i \pi-q^{\prime} a}=-\frac{1-Q}{1+Q} \approx-\exp (-2 Q),
$$

where

$$
Q=\sqrt{\frac{\cos ^{2}\left(\phi_{-}\right) r_{w b}^{2}-\cos ^{2}\left(\phi_{+}\right)}{\sin ^{2}\left(\phi_{+}\right)-\sin ^{2}\left(\phi_{-}\right) r_{w b}^{2}}} .
$$

In the case of small $r_{w b}$ we can expand $\phi_{+}$near the central point $k_{v}$ of the Bragg peak, where $\phi_{+}=\pi n / 2, \phi_{-}=\pi m / 2$ and $m<n$. This expansion gives

$$
Q \approx r_{w b} \sqrt{1-\left(x / x_{0}\right)^{2}},
$$

where $x=\left(k-k_{v}\right) / k_{v}$ and

$$
x_{0}=\frac{4}{\pi} r_{w b} \frac{k_{w}^{2} k_{b}^{2}}{k_{v}^{2}\left[n\left(k_{w}^{2}+k_{b}^{2}\right)-m\left(k_{w}^{2}-k_{b}^{2}\right)\right]} .
$$

The Darwin width $\Delta$ is determined by $x_{0}$. Larger the $m$ is, larger is $x_{0}$, so it is profitable to have $m=n-1$. With this $m$ the parameter $x_{0}$ is

$$
x_{0}=\frac{4}{\pi} r_{w b} \frac{k_{w}^{2} k_{b}^{2}}{k_{v}^{2}\left[2 n k_{b}^{2}+\left(k_{w}^{2}-k_{b}^{2}\right)\right]} .
$$

It is the largest for the smallest $n$. Thus the best choice for $n$ is $n=1$ and we finally get

$$
x_{0}=\frac{4}{\pi} r_{w b} \frac{k_{w}^{2} k_{b}^{2}}{k_{v}^{2}\left(k_{w}^{2}+k_{b}^{2}\right)} .
$$

Now we need to find the ends $k_{v e}$ of the Darwin table around $k_{v}$. They depend on the amount of deviation from total reflection that we can tolerate. If we tolerate $|R|^{2}=1$ $-2 \zeta$, then from Eqs. (4) and (15) it follows

$$
Q \geqslant-\ln (\zeta) / 4 N \text {. }
$$

From this we find

$$
\left(\frac{x}{x_{0}}\right)^{2} \leqslant 1-\left(\frac{\ln \zeta}{4 N r_{w b}}\right)^{2}
$$




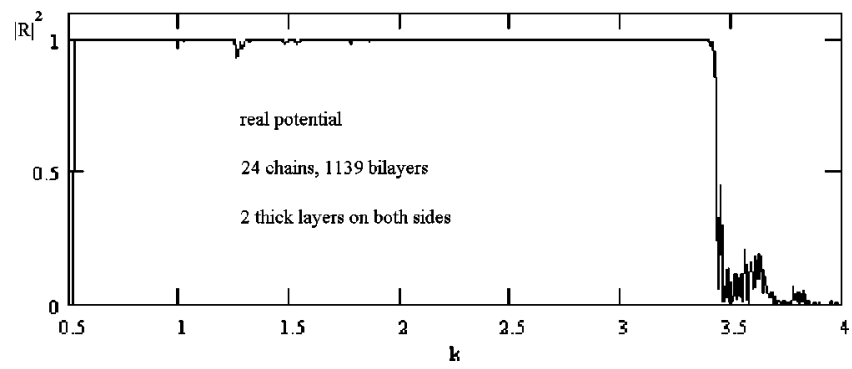

FIG. 6. Dependence of the reflection coefficient $|R|^{2}$ on $k$ for MS with real potentials. MS consists of 24 chains with different number of periods. Total number of bilayers is 1139 . There are two barriers of width $l_{0}=10$ on both sides of MS. Critical $k_{c}$ for the barrier is equal to 1 .

To find the optimal number $N$ of periods, we require the maximal effective width of $\delta k$ covered by a single period, i.e., we seek a maximum of $x / N$. The maximum corresponds to

$$
N=\frac{\ln \zeta}{2 \sqrt{2} r_{w b}}
$$

and

$$
\delta k=\left|k_{v e}-k_{v}\right|=k_{v} x_{0} \frac{1}{\sqrt{2}}=\frac{2 \sqrt{2} r_{w b} k_{w}^{2} k_{b}^{2}}{\pi k_{v}\left(k_{w}^{2}+k_{b}^{2}\right)} \approx \frac{\sqrt{2}}{\pi} k_{v} r_{w b},
$$

where $k_{w}, k_{b}$, and $r_{w b}$ are determined for $k=k_{v}$. If we tolerate $2 \zeta=1 \%$, then $N=1.9 / r_{w b}$.

Now, the interval $\Delta^{\prime}=2 \delta k=(2 \sqrt{2} / \pi) k_{v} r_{w b}$ around $k_{v}$ is closed and we can make the step to a new $k_{v}^{\prime}=k_{v}+\Delta^{\prime}$ and find a new periodic chain around the point $k_{v}^{\prime}$. In practice, we made steps $\Delta^{\prime}=2 \delta k / 1.2$ to ensure the overlapping of the intervals, because every next width $\delta k$ is a little bit lower than the preceding one.

In Fig. 6 the reflection coefficient $|R|^{2}$ is shown for MS consisting of 1139 bilayers with positions of the Bragg peaks chosen as prescribed above. The starting point is $k_{v}=1.18$ and the next one is $k_{v}=1.42$. They were found a little bit empirically. Both ends of the multilayer system are closed by thick layers $\left(l_{0}=10\right)$ with potential barrier, giving total reflection for $k<1$. We see that the reflection coefficient is almost perfectly equal to one for real potentials. The defect

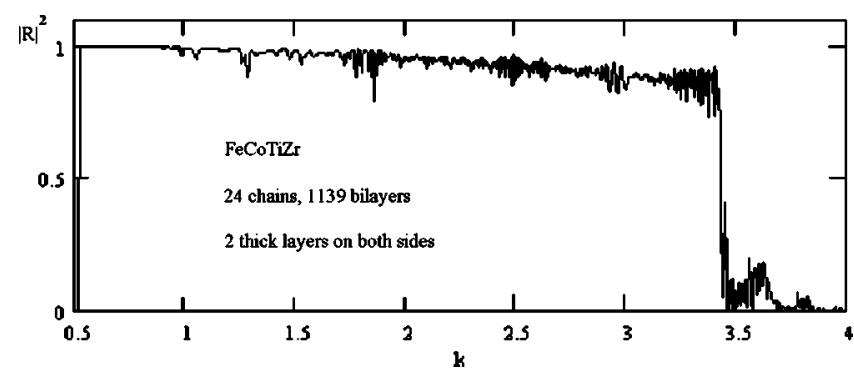

FIG. 7. Dependence of the reflection coefficient $|R|^{2}$ of FeCoTiZr MS on $k$. Parameters of MS are the same as for Fig. 6. Potentials include imaginary parts.

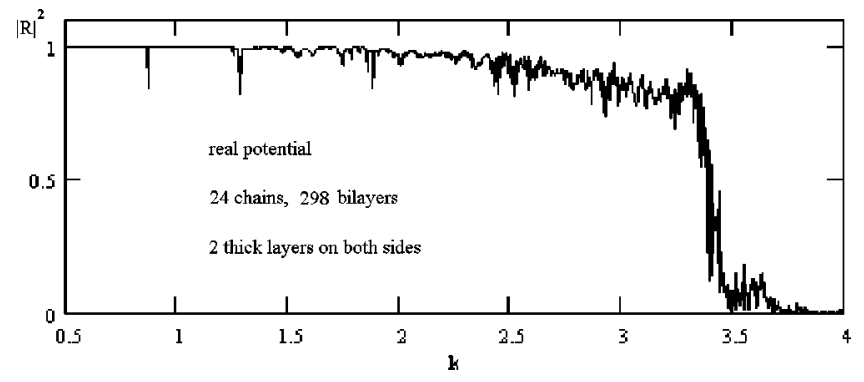

FIG. 8. Dependence of reflection coefficient $|R|^{2}$ of the same system as in Fig. 6 with number of periods in every periodic chain strongly decreased. Total number of bilayers is 298 .

seen on the reflection curve can be easily repaired by adding one more periodic chain with the Bragg peak at the position of this defect, or by slight shift of some $k_{v}$ toward a lower value.

In the development presented above, we did not take into account the imaginary part of the potential; however, formulas (2) - (5) and (10)-(12) are valid for arbitrary potentials, so in order to take into account losses or gains (in the case of active media) we need only substitute into $k_{w}$ and $k_{b}$ the complex potentials $u_{w, b}=u_{w, b}^{\prime}-i u_{w, b}^{\prime \prime}$, where minus sign means losses for $u^{\prime \prime}>0$. Of course, the number and widths of layers in periodic chains and the widths of the Bragg peaks are real numbers so, for them, we must use absolute magnitudes.

The result of calculations for FeCo-TiZr MS, which is similar for FeCo-Si, is shown in Fig. 7. Here the number of bilayers is the same as in Fig. 6, and we see that because of the imaginary part of the potentials the reflection coefficient deviates from unity. It means that our requirement (17) with small $\zeta$ is too strict and not necessary, because the imaginary part of the potentials makes us tolerant to stronger deviation of the Bragg reflection from unity. So we can strongly decrease the number of periods in every chain considerably.

In Fig. 8 we show how the reflection coefficient presented in Fig. 6 changes when the number of bilayers is decreased to 298. We see that now it becomes similar to that shown in Fig. 7. If we account for imaginary parts of the potentials then for the FeCo-TiZr MS with 298 bilayers we obtain the reflection coefficient shown in Fig. 9. We see that it is almost the same as that shown in Fig. 8, which means that imaginary parts of the potentials do not spoil it further.

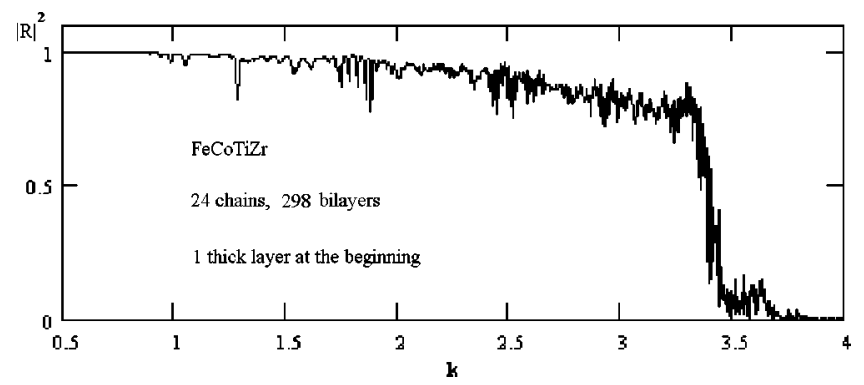

FIG. 9. Dependence of the reflection coefficient $|R|^{2}$ of FeCoTiZr MS on $k$. Parameters of MS are the same as for Fig. 7, but the number of bilayers is only 298 . 


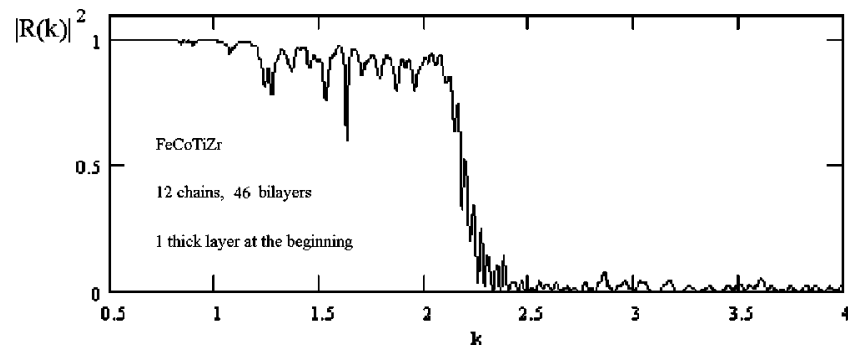

FIG. 10. Reflection of 46 bilayers of FeCo-TiZr system with account of losses. Parameters of layers are shown in Table I.

In the case we are satisfied with smaller increase of the critical angle, we need an even smaller number of layers. In Fig. 10 we show the reflection coefficient for FeCo-TiZr with only 46 bilayers. The parameters of these bilayers are shown in Table I.

\section{REFLECTION FROM THE SET OF CHAINS}

If we have two chains with reflection and transmission amplitudes $R_{N i}, T_{N i}(i=1,2)$, then the reflection amplitude $R_{21}$ from two chains from the left (the chain 1 is to the right of the chain 2) is

$$
R_{21}=R_{N 2}+\frac{T_{N 2}^{2} R_{N 1}}{1-R_{N 2} R_{N 1}} .
$$

Addition of the third chain to the left side gives the reflection amplitude

$$
R_{321}=R_{N 3}+\frac{T_{N 3}^{2} R_{21}}{1-R_{N 3} R_{21}}
$$

Four chains will have reflection amplitude $R_{4321}$ and so on. It is a simple algorithm to calculate reflection from all the chains, and at the end we must add a single wide barrier as shown in Fig. 11, which provides total reflection for all $k$ almost up to $k=1$. Because of finite width $l_{0}$ of the first barrier, its reflectivity drops near $k=1$. Indeed, the reflection from the barrier is

$$
r=r_{w b} \frac{1-Q_{0}}{1-r_{w b}^{2} Q_{0}}
$$

where

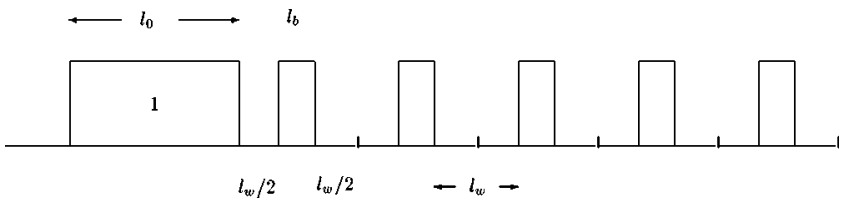

FIG. 11. Composition of MS with a wide barrier layer. The MS should contain a barrier of large width $l_{0}$ to provide total reflection almost up to $k=1$.

$$
r_{w b}=\frac{k_{w}-i k_{b}^{\prime}}{k_{w}+i k_{b}^{\prime}}, \quad k_{w}=\sqrt{k^{2}-u_{w}}, \quad k_{b}^{\prime}=\sqrt{1-k^{2}}
$$

and $Q_{0}=\exp \left(-2 l_{0} k_{b}^{\prime}\right)$.

Suppose, we tolerate, when $|r|^{2}=1-\zeta$. Near the critical point $k=1$, the reflection coefficient can be approximated as

$$
|r|^{2}=\frac{\left(1-Q_{0}\right)^{2}}{\left(1-Q_{0}\right)^{2}+16 k_{w}^{2} k_{b}^{2} Q_{0}} \approx 1-4 \frac{k^{2}}{l_{0}^{2}} .
$$

So, if we want to have $|r|^{2}$ to be everywhere in $0<k<1$ larger than $1-\zeta$, we must choose $l_{0}=2 / \sqrt{\zeta}$. In particular, for $\zeta=0.01$ we must choose $l_{0}=20$. In all the Figs. 6-9 we used the widths of the wide barriers at both ends of MS, or only at the beginning, equal to $l_{0}=10$. This parameter is not too critical, because though reflection of the smaller first barrier is a little bit less the losses in it are also less. So practically we have no gain, if we increase the totally reflecting layer, and we do not need two wide barriers. One barrier at the beginning of the MS is sufficient.

\section{ASYMMETRY OF THE PERIOD}

Above we considered the case when periods of periodical chains are symmetrical, i.e., the barrier of width $l_{b}$ is surrounded on both sides with wells of width $l_{w} / 2$, i.e., it is represented as a three layer. In practice, it is simpler to consider the period as a bilayer consisting of the well of width $l_{w}$ and the barrier of the width $l_{b}$. Such a period is not symmetrical. Its reflection from the left $r_{l}$ is not equal to reflection from the right, $r_{r}$, though transmissions from both sides are equal [see Eq. (5)]. The amplitudes $r_{l}$ and $r_{r}$ for the bilayer are

$$
r_{l}=e^{2 i k_{w} l_{w}} r_{w b} \frac{1-\exp \left(2 i k_{b} l_{b}\right)}{1-r_{w b}^{2} \exp \left(2 i k_{b} l_{b}\right)}=e^{i k_{w} l_{w} r},
$$

TABLE I. Parameters of 12 periodic chains with the reflection coefficient shown in Fig. 10. $k_{v}$ is the position of the Bragg peak, $l_{w}, l_{b}$, and $N$ are the widths of TiZr (or Si) and FeCo layers and number of bilayers, respectively, for the chain with the Bragg reflection centered at $k_{v}$. Total number of bilayers is 46. At the beginning of the MS there is a FeCo layer of thickness $l_{0}=8$, providing total reflection for $k$ up to 1 .

\begin{tabular}{lcccccccccccc}
\hline \hline $\begin{array}{l}\text { Param- } \\
\text { eters }\end{array}$ & 1 & 2 & 3 & 4 & 5 & 6 & 7 & 8 & 9 & 10 & 11 & 12 \\
\hline$k_{v}$ & 1.12 & 1.32 & 1.44 & 1.55 & 1.65 & 1.73 & 1.81 & 1.90 & 1.96 & 2.03 & 2.09 & 2.15 \\
$l_{b}$ & 3.11 & 1.84 & 1.5 & 1.331 & 1.20 & 1.11 & 1.04 & 0.98 & 0.93 & 0.89 & 0.86 & 0.82 \\
$l_{w}$ & 1.40 & 1.20 & 1.09 & 1.01 & 0.95 & 0.91 & 0.87 & 0.83 & 0.80 & 0.78 & 0.75 & 0.73 \\
$\mathrm{~N}$ & 3 & 3 & 3 & 3 & 3 & 4 & 4 & 4 & 4 & 5 & 5 & 5 \\
\hline \hline
\end{tabular}




$$
r_{r}=r_{w b} \frac{1-\exp \left(2 i k_{b} l_{b}\right)}{1-r_{w b}^{2} \exp \left(2 i k_{b} l_{b}\right)}=e^{-i k_{w} l_{w} r}
$$

where $r$ is the reflection amplitude (5) for the symmetrical period. With nonsymmetrical period expression (2) should be modified. For instance, reflection amplitude $R_{l}$ from the semiinfinite periodic potential beginning with the well is

$$
\begin{gathered}
\sqrt{\frac{r_{l}}{r_{r}}} \frac{\sqrt{\left(1+\sqrt{r_{r} r_{l}}\right)^{2}-t^{2}}-\sqrt{\left(1-\sqrt{r_{r} r_{l}}\right)^{2}-t^{2}}}{\sqrt{\left(1+\sqrt{r_{r} r_{l}}\right)^{2}-t^{2}}+\sqrt{\left(1-\sqrt{r_{r} r_{l}}\right)^{2}-t^{2}}} \\
=e^{i k_{w} l_{w} \frac{\sqrt{(1+r)^{2}-t^{2}}-\sqrt{(1-r)^{2}-t^{2}}}{\sqrt{(1+r)^{2}-t^{2}}+\sqrt{(1-r)^{2}-t^{2}}},}
\end{gathered}
$$

or it is $\exp \left(i k_{w} l_{w}\right) R$, where $R$ is the symmetrical amplitude given by Eq. (2). The reflection from semi-infinite periodic potential beginning with the barrier will be $\exp \left(-i k_{w} l_{w}\right) R$, i.e., asymmetry of $r$ is inherited by $R$.

Equation (3) for the Bloch phase factor does not change, because instead of $r^{2}$ it contains $r_{l} r_{r}$, which is identical to $r^{2}$. Now it is easy to understand that reflection of a finite number periods $R_{N}$ for asymmetrical period will change in the same way as $R$, i.e., for reflection from the left and right we have $R_{N l, r}=\exp \left( \pm i k_{w} l_{w}\right) R_{N}$, where $R_{N}$ denotes the reflection amplitude for symmetrical period.

We now need to see what happens when we stack two nonsymmetrical chains. For that we need to generalize expression (1) for nonsymmetrical potentials 1 and 2 shown in Fig. 1. This generalized expression is

$$
R_{l 12}=R_{l 1}+T_{1}^{2} \frac{R_{l 2}}{1-R_{r 1} R_{l 2}}
$$

where indices $l, r$ denote reflection from the left and right, respectively.

Taking into account this generalization, we represent Eq. (21) in the form

$$
R_{l 21}=e^{i k_{w} l_{w 2}}\left[R_{N 2}+\frac{T_{N 2}^{2} \exp \left(i k_{w}\left[l_{w 1}-l_{w 2}\right]\right) R_{N 1}}{1-R_{N 2} \exp \left(i k_{w}\left[l_{w 1}-l_{w 2}\right]\right) R_{N 1}}\right],
$$

where asymmetry is explicitly represented by the factor $\exp \left(i k_{w}\left[l_{w 1}-l_{w 2}\right]\right)$. It is easy to prove that if the chain 1 at some $k$ gives total reflection, i.e., $R_{N 1}=\exp (i \chi)$, then inclusion of chain 2 will not destroy this total reflection, i.e., $R_{l 21}$ for these $k$ is also a unit complex number: $R_{l 21}=\exp \left(i \chi^{\prime}\right)$. Indeed, taking into account relations (9), which are valid for $R_{N}$ and $T_{N}$, we can transform Eq. (26) as follows

$$
\begin{aligned}
R_{l 21}= & -e^{i k_{w} l_{w 1}+i \chi+2 i \phi_{2}} \\
& \times \frac{1-\left|R_{N 2}\right| \exp \left(-i k_{w}\left[l_{w 1}-l_{w 2}\right]-i \phi_{2}-i \chi\right)}{1-\left|R_{N 2}\right| \exp \left(i k_{w}\left[l_{w 1}-l_{w 2}\right]+i \phi_{2}+i \chi\right)},
\end{aligned}
$$

where $\phi_{2}$ is the phase of the amplitude $R_{N 2}$. Since the last factor is of the form $\exp (i \psi)$ the whole $R_{l 21}$ is also of the form $\exp \left(i \chi^{\prime}\right)$ that corresponds to the total reflection.

Of course, all these relations are precise only for real potentials. The imaginary part of the potentials gives a correction to them, and the smaller is the imaginary part, the smaller is the correction.

\section{SIMILARITY OF ALL THE MS SYSTEMS}

All the MS can be represented as a system with barriers of height 1 and wells of height 0 . Indeed, if in a practical system barriers have the potential $u_{b}$, and wells the potential $u_{w}$, then the potential step between the well and the barrier is $u_{b}-u_{w}$, and we can normalize this difference to unity, and take as a unit length the critical wavelength $\lambda / 2 \pi$ $=\hbar / \sqrt{2 m\left(u_{b}-u_{w}\right)}$. Hence, calculations for all the practical systems are the same. The only difference is that at the end we need to include the reflection amplitude from the potential step from vacuum to the well. This potential step is now a normalized potential $\tilde{u}_{w}=u_{w} /\left(u_{b}-u_{w}\right)$. If the reflection amplitude from MS without this correction is $R$, then after correction it will be

$$
r_{0 w}+\frac{\left(1-r_{0 w}^{2}\right) R}{1+r_{0 w} R}
$$

where

$$
r_{0 w}=\frac{k-\tilde{k}_{w}}{k+\tilde{k}_{w}}, \quad \tilde{k}_{w}=\sqrt{k^{2}-\tilde{u}_{w}} .
$$

In our calculations we did not make this correction and took $u_{w}=0$ having in mind reflection of neutrons from inside of a Si crystal, as was proposed in Ref. [20].

We applied our method to practical systems and considered only 24 chains, though it is not critical. With these chains we are able to increase the critical angle $\approx 3.5$ times. If we want only to double the critical angle, we need only 12 chains. Their parameters are presented in Table I. The first row shows the points $k_{v}$ that are centers of the Bragg peaks. The first number $k_{v}=1.12$ was chosen somewhat ad hoc. Next two rows show the width of the wells $l_{w}$ and barriers $l_{b}$ for those $k_{v}$ and the last row shows the number of periods in every chain.

Imaginary parts for potentials of practical systems were normalized to the difference of real parts of $u_{b}-u_{w}$. Thus for the FeCo-Si system in which $\mathrm{Si}$ are wells with $u_{w}$ $=54.4-i 6.25 \times 10^{-4} \mathrm{neV}$ and FeCo are barriers with $u_{b}$ $=330.7-i 6.40 \times 10^{-2} \mathrm{neV}$, the normalized potentials are $u_{b}=1-i 2 \times 10^{-4}$ and $u_{w}=0-i 2.3 \times 10^{-6}$.

In the FeCo-TiZr system the normalized imaginary part of $\mathrm{FeCo}$ is $\approx 3 \times 10^{-4}$ and that of $\mathrm{TiZr} \approx 1 \times 10^{-4}$. The main effect of losses comes from the imaginary part of FeCo, so the results of calculations for practical systems with $\mathrm{Si}$ and TiZr give nearly the same result. 


\section{CONCLUSION}

We presented a method of calculating properties of a supermirror with a high critical angle of total reflection. We suppose that our method has some advantage, because it is analytical and therefore amenable to optimization techniques. In the cases presented here, change of parameters $\delta l_{b}$ and $\delta l_{w}$ from chain to chain is sufficiently large and therefore is less prone to errors related to the technology of layers preparation. There is only a title change of parameters compared to the common approach of building multilayer systems, when the parameters change almost continuously, and $\delta l_{b}, \delta l_{w}$ become lower than a monolayer. Such a small change of width is almost impossible to control.

We want also to add that though our analytical method is very good for analysis, actual calculation of the reflection coefficient, after all the parameters are defined from our analytical method, can be performed numerically with the matrix method.

We have shown here how to prepare MS by increasing the range of total reflection step by step. However, it is possible to proceed differently. We can put one bilayer on a substrate and calculate its reflection. Then we put another bilayer with parameters scanned in some intervals and choose parameters, which give the larger increase of the reflectivity. Then we look for parameters of third bilayer and so on. If we do not restrict thickness of layers, we can get with 200 bilayers a good reflectivity as shown in Fig. 12 for some model system with $u_{w}=-1$ even for interval $k=4 k_{c} \cdot{ }^{1}$ However, in these bilayers some thicknesses are of the order 0.1 of interatomic distance. It is clear that it is impossible to achieve a good homogeneity for such thicknesses. We can restrict thicknesses to some values when scanning in the parameter space. It may give a not-so-perfect multilayer system with a smaller

${ }^{1}$ If we normalize to unity the sum $u_{b}+\left|u_{w}\right|$, then the increase in Fig. 12 is not 4 , but $\sqrt{17 / 2}=2.9$.

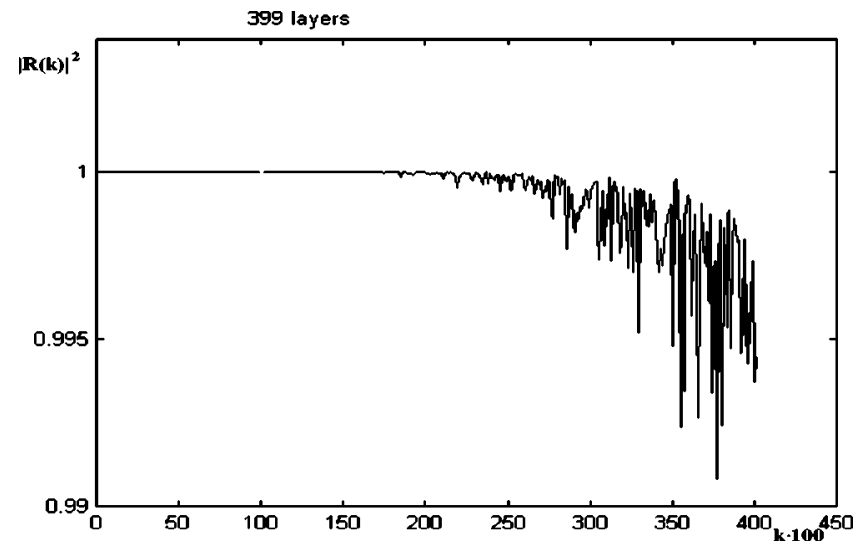

FIG. 12. Reflection of 399 layers for a model system with $u_{w}$ $=-1$.

number of layers but with a good enough reflectivity in a wide enough interval of $k$.

Though this trial-and-error method may give a tolerable reflection with a smaller number of periods, our step-by-step method is more promising for the technology of MS preparation. It is evident that in order to have good interfaces the thicknesses of a layers must be equal to integer number of monolayers. If a layer contains a fraction of monolayer, its surface will never be flat, even if it is evaporated on a substrate with an ideal surface.

When we know the exact thickness of a single monolayer we can calculate reflection amplitudes for layers with integer number of monolayers. Comparison of calculations with really obtained reflectivities in a wide range of energies provides a good control for development of technology for MS preparation.

We considered here only the reflection of neutrons from MS. This method can also be applied to $x$ rays. In that case, we can reliably account for the imaginary part of the potentials, which is large compared to that of optical potentials for neutrons.
[1] V.F. Turchin, Atomnaya Energiya 22, 119 (1967).

[2] V.F. Turchin, Phys. At. Nucl. 60, 1946 (1997).

[3] F. Mezei, Commun. Phys. (London) 1, 81 (1976).

[4] J.B. Hayter and H.A. Mook, Appl. Cryst. 22, 35 (1989).

[5] C.G. Darwin, Philos. Mag. 27, 675 (1914).

[6] G.H. Godfrey, Aust. J. Phys. 10, 1 (1957).

[7] O. Shärpf, Physica B 174, 514 (1991).

[8] P. Böni, D. Clemmens, M.S. Kumar, and C. Papas, Physica B 267-268, 320 (1999).

[9] A. Ioffe, P. Fischer, T. Krist, and F. Mezei, J. Phys. Soc. Jpn., Suppl. A 65, 80 (1996).

[10] A. Schebetov, J. Phys. Soc. Jpn., A 65, 195 (1996).

[11] S. Itoh and M. Takeda, J. Phys. Soc. Jpn., Suppl. A 65, 199 (1996).
[12] M. Takeda, K. Kurahashi, Y. Endoh, and S. Itoh, J. Phys. Soc. Jpn., Suppl. A 65, 234 (1996).

[13] T. Kawai, J. Phys. Soc. Jpn., Suppl. A 65, 230 (1996).

[14] K. Al Usta, K. N'guy, A. Menelle, and F. Samuel, J. Phys. Soc. Jpn., Suppl. A 65, 249 (1996).

[15] S. Basu, J. Phys. Soc. Jpn., Suppl. A 65, 289 (1996).

[16] V.K. Ignatovich, Am. J. Phys. 57, 873 (1989).

[17] F. Radu and V.K. Ignatovich, Physica B 267, 175 (1999).

[18] V.K. Ignatovich, Physica B 175, 33 (1991).

[19] V.K. Ignatovich, Physics of Ultracold Neutrons (UCN) (Clarendon Press, Oxford, 1990).

[20] V.G. Syromyatnikov, A.F. Schebetov, and Z.N. Soroko, Nucl. Instrum. Methods Phys. Res. A 324, 401 (1993). 